\title{
Fully Noncontact Wave Propagation Imaging in an Immersed Metallic Plate with a Crack
}

\author{
Jung-Ryul Lee, ${ }^{1}$ Jae-Kyeong Jang, ${ }^{1}$ and Cheol-Won Kong ${ }^{2}$ \\ ${ }^{1}$ Department of Aerospace Engineering and LANL-CBNU Engineering Institute Korea, Chonbuk National University, \\ 664-14 Deokjin-dong, Deokjin-gu, Jeonju, Chonbuk 561-756, Republic of Korea \\ ${ }^{2}$ Structures and Materials Department, Korea Aerospace Research Institute, Daejeon 305-333, Republic of Korea \\ Correspondence should be addressed to Jung-Ryul Lee; leejrr@jbnu.ac.kr
}

Received 12 February 2013; Accepted 11 June 2013; Published 5 May 2014

Academic Editor: Gyuhae Park

Copyright (C) 2014 Jung-Ryul Lee et al. This is an open access article distributed under the Creative Commons Attribution License, which permits unrestricted use, distribution, and reproduction in any medium, provided the original work is properly cited.

\begin{abstract}
This study presents a noncontact sensing technique with ultrasonic wave propagation imaging algorithm, for damage visualization of liquid-immersed structures. An aluminum plate specimen $(400 \mathrm{~mm} \times 400 \mathrm{~mm} \times 3 \mathrm{~mm})$ with a $12 \mathrm{~mm}$ slit was immersed in water and in glycerin. A $532 \mathrm{~nm}$ Q-switched continuous wave laser is used at an energy level of $1.2 \mathrm{~mJ}$ to scan an area of $100 \mathrm{~mm}$ $\times 100 \mathrm{~mm}$. A laser Doppler vibrometer is used as a noncontact ultrasonic sensor, which measures guided wave displacement at a fixed point. The tests are performed with two different cases of specimen: without water and filled with water and with glycerin. Lamb wave dispersion curves for the respective cases are calculated, to investigate the velocity-frequency relationship of each wave mode. Experimental propagation velocities of Lamb waves for different cases are compared with the theoretical dispersion curves. This study shows that the dispersion and attenuation of the Lamb wave is affected by the surrounding liquid, and the comparative experimental results are presented to verify it. In addition, it is demonstrated that the developed fully noncontact ultrasonic propagation imaging system is capable of damage sizing in submerged structures.
\end{abstract}

\section{Introduction}

Lamb waves are useful for the detection of damages in thin sheet materials and tubular properties. Extensive developments in the application of the Lamb wave provide a foundation for the inspection of many products in the aerospace, pipe, pipeline, and transportation industries. Lamb waves are composed of a combination of two fundamental modes: symmetric and antisymmetric. For each of these modes, their velocity (phase or group) of Lamb waves varies with frequency; in other words, they are all dispersive. And, their energy is spread in time and space as it propagates. Hence, as the distance increases, the signal duration increases and the peak amplitude decreases. Also, attenuation is of concern in specimens immersed in liquid, because of leaky Lamb waves. For example, when guided Lamb waves are propagating in a plate that is placed in vacuum or in air, both plate surfaces are considered to be untrammeled. But, if one or both of the surfaces are in contact with liquid, the guided plate waves become leaky Lamb waves, because the energy of the wave leaks to the adjacent liquid.

Ultrasonic waves are increasingly being investigated for nondestructive evaluation (NDE) and structural health management (SHM) of engineering systems, because they can propagate over long distances and cover relatively large areas of thin plates. They can travel comparatively large distances with little attenuation and offer the advantage of exploiting one or more of the phenomena associated with transmission, reflection, scattering, and mode conversion. A few studies have been reported concerning the use of ultrasonic waves for underwater structures.

$\mathrm{Na}$ and Kundu [1] investigated the feasibility of flexural cylindrical guided waves for inspecting the mechanical defects of underwater pipes, using a transducer holder and its coupling mechanism. Mijarez et al. [2] developed a system composed of a waterproof transmitter and a seawateractivated battery package, to monitor the tubular crossbeam members used in offshore steel structures. Chen et 


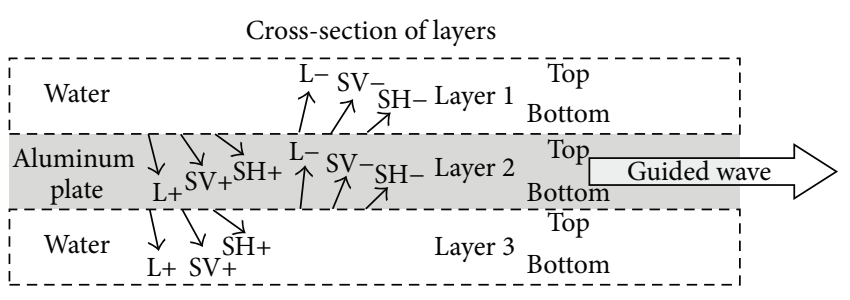

FIGURE 1: Geometry of a three-layer flat plate system showing the partial waves in each layer ( $\mathrm{L}+-_{-} \mathrm{SV}_{+-}$, and $\mathrm{SH+-}$ ) that combine to produce a guided wave [7].

al. [3] proposed a damage identification approach capitalizing on the fundamental asymmetric Lamb wave. Aristégui et al. [4] presented wave propagation characteristics of pipes with fluid loading on both inside and outside of the pipe, which was affected by viscosity of the media. A noncontact approach was also used for the generation and detection of Lamb waves, because of the circumstance of the target to be inspected. Rizzo et al. [5] presented a SHM technique using a hybrid laser/immersion transducer system for the detection of damage in submerged structures. Xu et al. [6] presented a comparison between theoretical predictions and experimental results, to consistently reveal the propagation properties of Lamb waves on a specimen that was in contact with different liquids on both of its surfaces, using laser generation and laser Doppler vibrometer (LDV) sensing.

In this paper, variation of certain mode is analyzed, to evaluate the size and shape of the damage for a test specimen in different boundary conditions: free plate and immersed plate with liquid. A laser ultrasonic propagation imaging (UPI) system capable of fully noncontact inspection of immersed structures is developed, through the modification of the sensor-contact UPI system, based on piezoelectric sensing. In addition, this study visualizes that the Lamb wave propagation characteristics, such as dispersion and attenuation, are affected by liquid and comparative experimental results are presented to verify it.

\section{Lamb Waves Propagation in Immersed Thin Plates}

2.1. Lamb Waves. A wave mode is defined as the manner of particle movement during wave propagation. In a solid material, particles can oscillate in four primary modes: longitudinal waves, shear transverse waves, surface waves, and plate waves in thin materials. These waves occur as a result of the elastic deformation of material. Lamb waves are complex vibration waves that propagate parallel to the specimen surface. Propagation of Lamb waves depends on the density and the elastic material properties of a component. They are also affected by the test frequency and thickness of structure.

Lamb waves can be generated in a plate with free boundaries, with an infinite number of modes, for both symmetric and antisymmetric displacements within the layer.

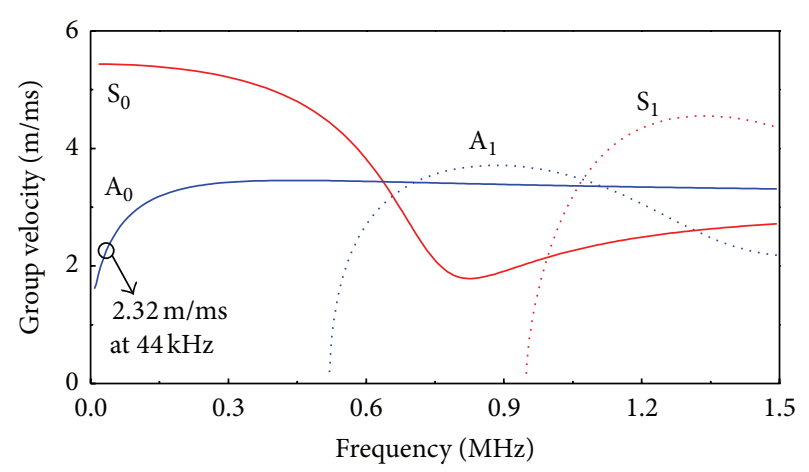

(a)

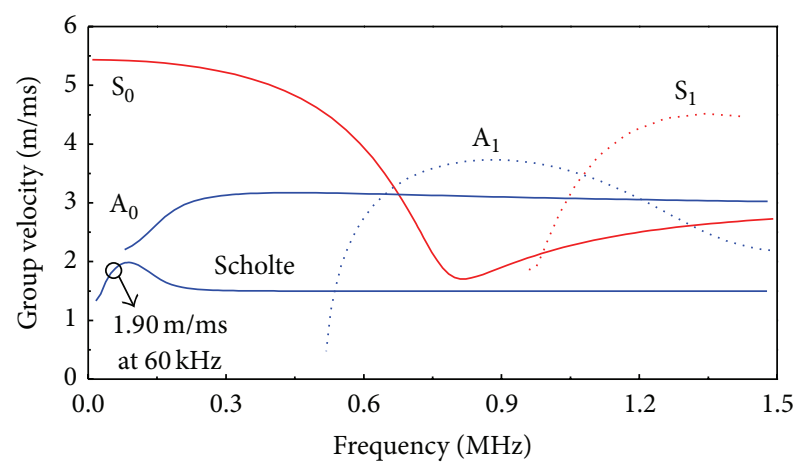

(b)

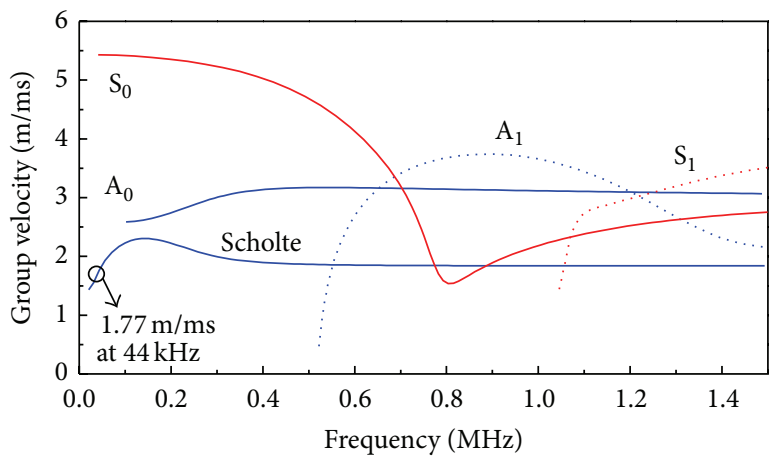

(c)

FIGURE 2: Group velocity dispersion curves of $3 \mathrm{~mm}$ thick aluminum plate: (a) free plate, (b) immersed in water, and (c) immersed in glycerin.

It is stipulated that the symbols $S_{i}$ and $A_{i}(i=0,1, \ldots)$ stand for the symmetric and antisymmetric modes, respectively, and the subscript $i$ implies the order of the mode. Symmetric Lamb waves move in a symmetric fashion about the median plane of the plate. Wave motion in the symmetrical mode is most efficiently produced when the exciting force is parallel to the plate. The antisymmetric Lamb wave mode is often called the "flexural mode" because a large portion of the motion moves in a normal direction to the plate, and a little motion occurs in the direction parallel to the plate. In the laser pulse excitation used in this paper, the magnitude of $S_{i}$ modes (in-plane motion) is normally smaller than that of $A_{i}$ modes. 


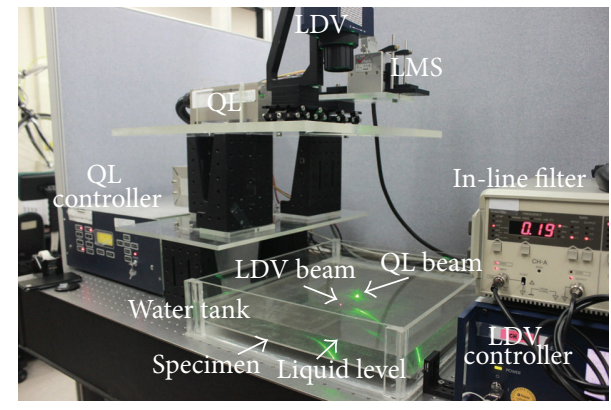

(a)
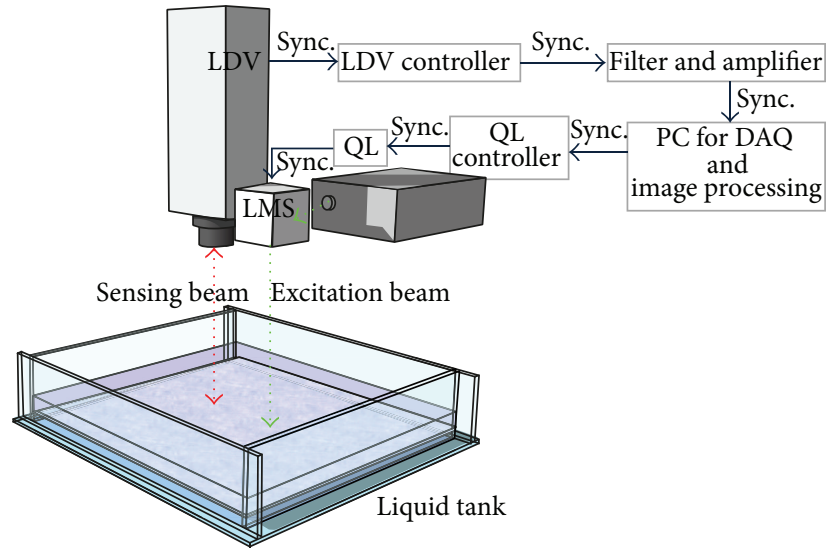

LMS: laser mirror scanner QL: Q-switched laser
LDV: laser Doppler vibrometer DAQ: data acquisitions

(b)

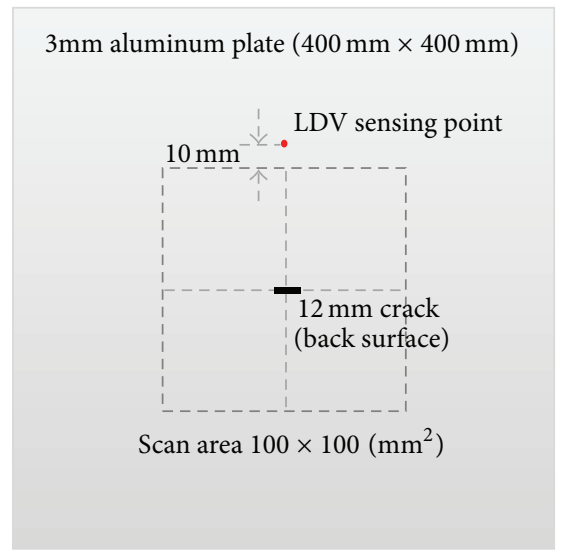

(c)

FIGURE 3: Experimental setup: (a) photo and (b) schematic of the UPI system for submerged structural inspection and (c) specimen with a back surface crack for immersion.

2.2. Wave Propagation with Attenuation in Submerged Plates. When a plate is submerged in an infinite liquid, the Lamb wave propagation energy will be leaked into the liquid. This wave is called a leaky Lamb wave. For example, when a plate is immersed in a liquid, such as water or glycerin, symmetric modes will mostly be retained in the plate, because it is difficult for in-plane particle motion to cross the plate-liquid interface. However, as the antisymmetric modes mostly have out-of-plane displacements, they will leak into the fluid. The leaky Lamb waves behave differently from Lamb waves in free solid. For instance, the dispersion equations associated with the first antisymmetric mode $\left(A_{0}\right)$ show a large discrepancy between the free solid and fluid-coupled solid [8].

In this research, the sample geometry of a three-layer flat and thin plate system was considered, as shown in Figure 1. According to [7], the partial waves are assembled by matching the boundary conditions at each layer. At a certain frequency, wave number, and attenuation combination, these partial waves combine to form a guided Lamb wave, which propagates down to the longitudinal direction. Here, L+-, $\mathrm{SV}+-$, and $\mathrm{SH}+-$ stand for longitudinal waves, shear vertical waves, and shear horizontal waves, respectively. In addition, + denotes the downward direction and the - denotes the upward direction of the plate case. We did not consider the $\mathrm{SH}+-$ waves, because they were difficult to generate by laser excitation.

2.3. Dispersion Curves in Different Boundary Conditions. If the plate is surrounded by liquid or solid, wave attenuation also occurs due to the leakage of bulk waves into the medium surrounding the waveguide. In this study, to investigate the acoustic properties of the leaky Lamb wave, dispersion curves for a $3 \mathrm{~mm}$ thick aluminum plate in three different cases were calculated; free plate and immersed plate in water and in glycerin, as presented in Figure 2. In an aluminum plate immersed in water or glycerin, the $A_{0}$ mode disappeared, and its energy was converted to Scholte wave below about $100 \mathrm{kHz}$. Group velocity of the $A_{0}$ mode became slower than that in the free aluminum plate. The Scholte wave is an interface wave between a liquid medium and a solid medium and decreases exponentially away from the surface, into the liquid medium [9]. 


\section{Noncontact Laser Inspection of Immersed Plates}

3.1. Ultrasonic Propagation Imaging System for Submerged Structural Inspection. A photo and schematic of the ultrasonic propagation imaging (UPI) system to inspect submerged structures are shown in Figures 3(a) and 3(b). The system was constructed with a laser Doppler vibrometer (LDV), in-line signal conditioner with filters and amplifiers, a personal computer (PC) with a data acquisition and signal processing platform, and a Q-switched solid-state diode pumped laser (QL). The laser pulses were generated by Qswitching technique in $\mathrm{QL}$, at a pulse repetition rate of $200 \mathrm{~Hz}$. The laser beam with a wavelength of $532 \mathrm{~nm}$ and energy of $1.2 \mathrm{~mJ}$ was directed to a laser mirror system (LMS). The laser beam was reflected toward the target specimen by a pair of laser mirrors in the LMS.

As shown in Figure 3(c), an aluminum plate $(400 \mathrm{~mm} \times$ $400 \mathrm{~mm} \times 3 \mathrm{~mm}$ ) with a $12 \mathrm{~mm}$ long and $2 \mathrm{~mm}$ deep artificial crack in the opposite surface was used as the specimen. The scanning area and interval were $100 \mathrm{~mm} \times 100 \mathrm{~mm}$ and $0.5 \mathrm{~mm}$, respectively and thus it took 202 seconds to scan the area. The $633 \mathrm{~nm}$ sensing laser beam of the LDV was impinged at a point $10 \mathrm{~mm}$ above the square scanning area. The typical reflection film was not used on the plate surface.

Ultrasonic sensing using the LDV is based on the detection of the Doppler shift of the laser light. The Doppler shift refers to the frequency shift of the light that is reflected back from the vibrating object to the source. The signal processing platform visualizes the wave propagation in the immersed specimen, using the basic ultrasonic wave propagation imaging (UWPI) algorithm. The UWPI is a visualization technology of propagation for in-plane guided wave or through-thethickness wave, in the time or frequency domain, based on $3 \mathrm{D}$ data processing [10].

In the experiments, when the laser beam impinged the surface of the immersed target specimen, an ultrasonic wave was created at the affected point. The wave was propagated over the specimen and reached the LDV. The time domain ultrasonic wave measured by the LDV was amplified and band-pass filtered between $40 \mathrm{kHz}$ and $140 \mathrm{kHz}$ and then stored in the PC. The experimental case studies were performed with three different cases: free plate and immersed plate in water and in glycerin, as shown in Figure 4.

\subsection{Ultrasonic Wave Propagation Imaging in Submerged} Plates. The proposed system for submerged structural inspection was able to generate UWPI video clips within $1 \mathrm{~s}$ after scanning. Figure 5 shows the freeze-frame at $53.5 \mu \mathrm{s}$ extracted from the video clip, as the UWPI result in the free plate. On the other hand, Figures 6 and 7 show the freeze-frames at $61 \mu$ s and $63 \mu$ s extracted from the videos as the UWPI results in the water- and glycerin-immersed plates, respectively. Since the proposed system was designed with both remote excitation and sensing laser beams capable of penetrating the liquid, the UWPI results were successfully obtained. All the freeze-frames in Figures 5-7 were taken at the moments when the maximum ultrasonic amplitudes

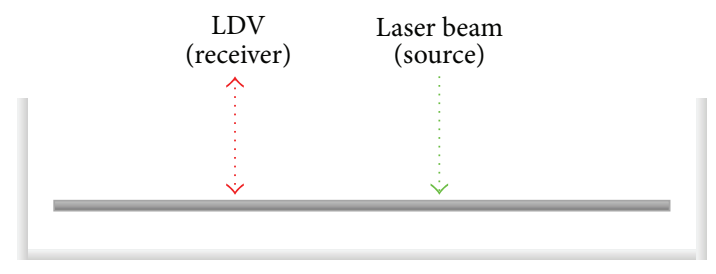

(a)

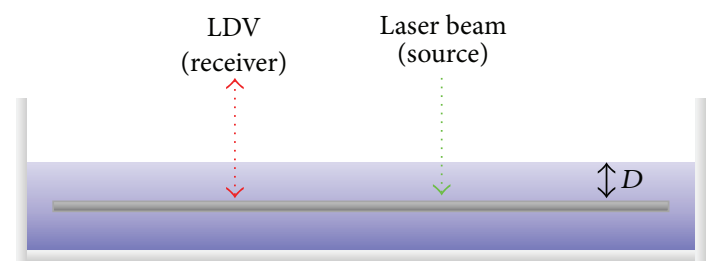

(b)

FIGURE 4: Experimental model in different conditions: (a) free plate in air and (b) immersed plate.

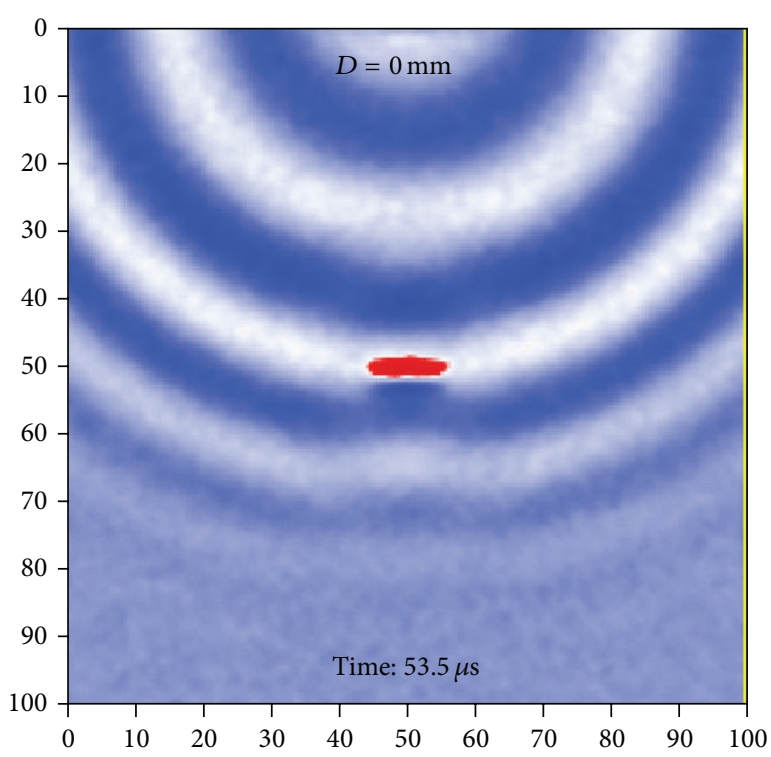

FIGURE 5: Snapshot at $53.5 \mu$ s taken from the ultrasonic wave propagation video clip in the free plate.

at the crack location appeared. As a result, it has been verified that the velocity of wave propagation was reduced in the immersed plates, due to the high densities of the surrounding fluid (air $=1 \mathrm{~kg} / \mathrm{m}^{3}$, water $=1,000 \mathrm{~kg} / \mathrm{m}^{3}$, and glycerin $=1,258 \mathrm{~kg} / \mathrm{m}^{3}$ ). In addition, as shown in Figures 6 and 7 , including the results for different depth $(D)$ of the liquid, such as $10 \mathrm{~mm}, 40 \mathrm{~mm}$, and $70 \mathrm{~mm}$, the propagation time was not affected by the tested depth of the water. In other words, even $10 \mathrm{~mm}$ deep surrounding liquid can be considered as an infinite surrounding medium.

In addition to the successful UWPI in the submerged specimens, the back surface crack that the water- or glycerinimmersed specimen encompasses was visualized in the form of sudden phase change and high peak-to-peak amplitude at the crack location, $(50,50)$, in the freeze-frames of 


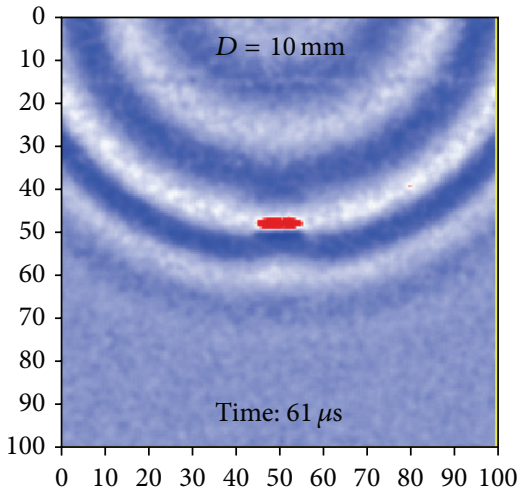

(a)

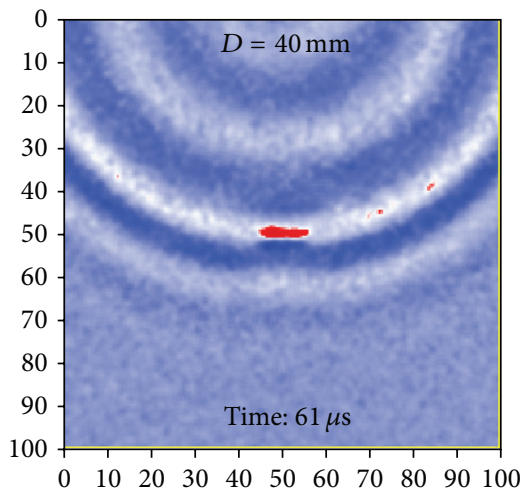

(b)

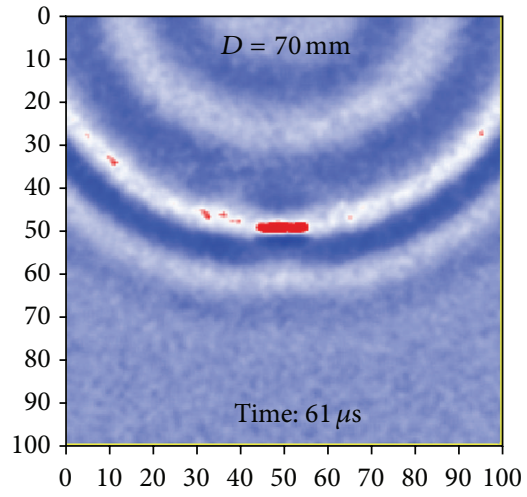

(c)

Figure 6: Ultrasonic wave fields at $61 \mu \mathrm{s}$ in the water-immersed plate according to the water depth: (a) $D=10 \mathrm{~mm}$, (b) $40 \mathrm{~mm}$, and (c) $70 \mathrm{~mm}$ (the same color scale for (a) to (c)).

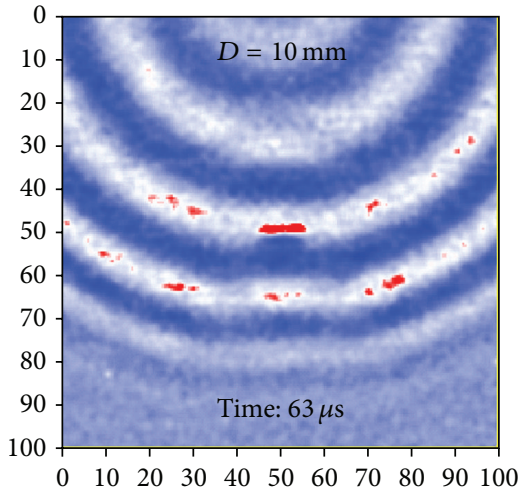

(a)

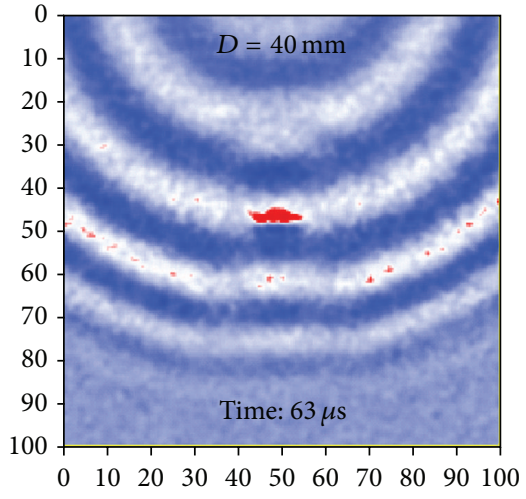

(b)

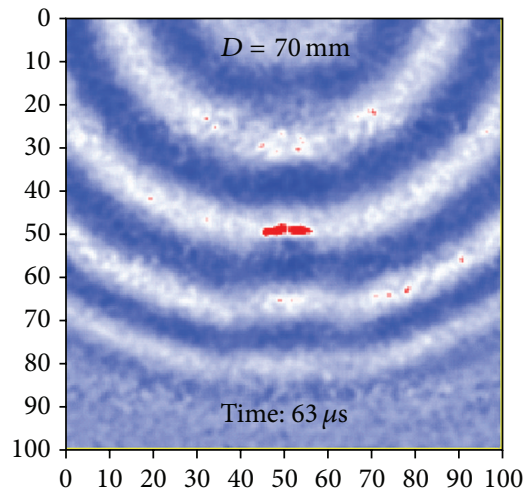

(c)

FIGURE 7: Ultrasonic wave fields at $63 \mu$ in the glycerin-immersed plate according to the glycerin depth: (a) $D=10 \mathrm{~mm}$, (b) $40 \mathrm{~mm}$, and (c) $70 \mathrm{~mm}$ (the same color scale for (a) to (c)).

Figures 7 and 8, respectively. However, in contrast to the free plate, a low SNR problem was identified in the immersed conditions, because of the effect of the surrounding liquid. As studied in Section 2.2, the surrounding liquid caused leaky Lamb wave occurrence. As concluded in Figures 6 and 7 , the energy losses within the liquid of excitation and the sensing laser beams themselves were negligible, because when the depth of liquid was increased, the SNR did not change considerably. For this reason, repeat scanning technology [11] was implemented in the proposed system for the submerged structural inspection, to increase the SNRs to be similar to the SNR level in the structure in air. The repeat scanning technique can play an important role in realworld submerged structural inspection. In the experiments, the scanning area was repeatedly scanned 10 times. The ten signals obtained at each scanning grid point were averaged, and the averaged waveforms were used as the input data for the UWPI algorithm [12] to generate the ultrasonic wave propagation video clips.

As compared between Figures 6(a) and 8(b) and between Figures $7(\mathrm{a})$ and $8(\mathrm{c})$, the respective SNRs under the water- and glycerin-immersed plate conditions were highly improved. The SNR changes before and after the repeat scanning are summarized in Table 1, where the SNR was evaluated in the way presented in Figure 9. On the other hand, the SNR improvement in the free plate by repeat scanning was not considerable, because the original raw signals already had low noise level. As compared between Figures 5, 8(b), and 8(c), the repeat scanning now made the SNRs in the water- and glycerin-immersed plate conditions reach similar levels to that in the free plate.

Figure 10 shows the amplitude distributions along the wavefront ( $s$-axis) at the moments of the maximum ultrasonic amplitude in the crack location, as indicated in Figure 8. And, the $s$-axis is formed along the same distance from the sensor location. The artificial crack length of $12 \mathrm{~mm}$ was estimated at $11.54 \mathrm{~mm}$ in the free plate, as shown in Figure 10(a). As also presented in Figures 10(b) and 10(c), the $12 \mathrm{~mm}$ long crack was evaluated by the proposed system as $11.29 \mathrm{~mm}$ in the water-immersed plate and $10.87 \mathrm{~mm}$ in the glycerin-immersed plate. These results were represented by two standard deviations away from the noise mean.

Figure 8 shows the spatial domain freeze-frames of the UWPI videos, while Figure 11 shows the time domain signals 


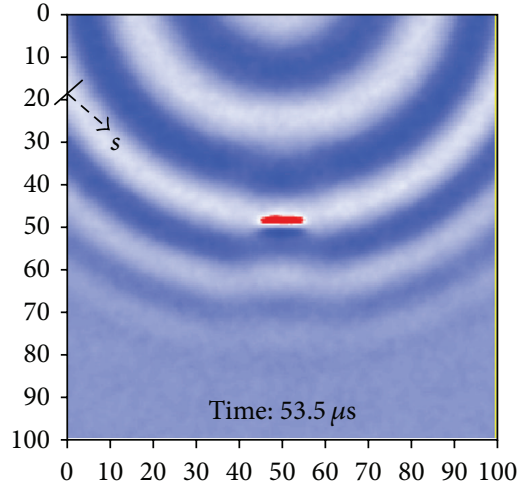

(a)

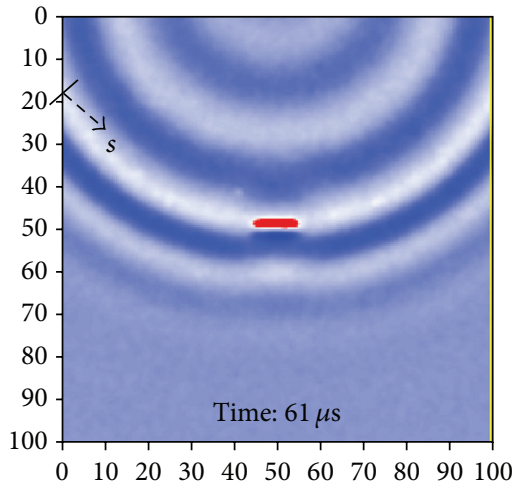

(b)

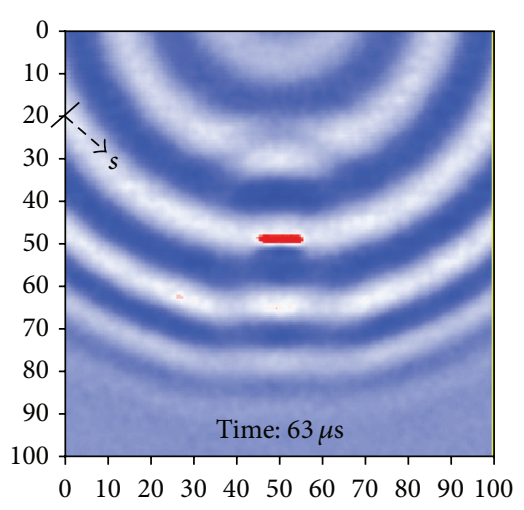

(c)

FIGURE 8: Ultrasonic wave fields obtained by the repeat scanning method (10 times): (a) free plate, (b) immersed plate in water $(D=10 \mathrm{~mm})$, and (c) immersed plate in glycerin $(D=10 \mathrm{~mm})$.

TABLE 1: SNR comparison related to immersion and repeat scanning.

\begin{tabular}{lccc}
\hline Number of repeat scanning & \multicolumn{2}{c}{$\begin{array}{c}\text { Signal-to-noise ratio (dB) } \\
\text { Water-immersed plate }\end{array}$} & Glycerin-immersed plate \\
\hline 1 & Free plate & 22.67 & 20.71 \\
10 & 26.01 & 28.23 & 26.18 \\
\hline
\end{tabular}

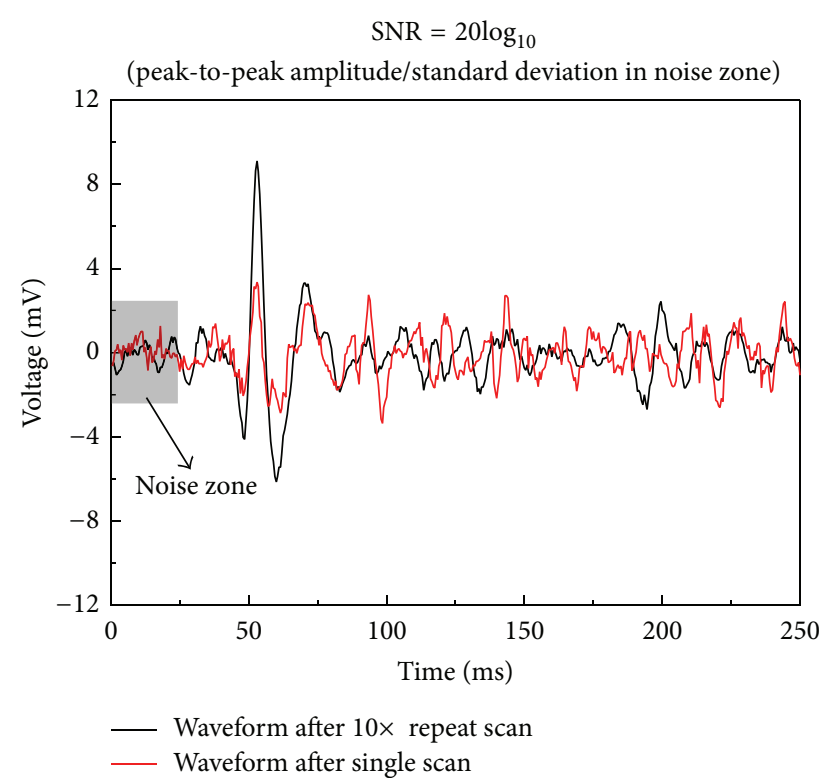

Figure 9: Typical time domain waveforms extracted at (20 and 25) in Figures 6(a) and 8(b) for SNR evaluation (waveform comparison after single scan and $10 \times$ repeat scan for water-immersed plate condition) and definition for SNR calculation.

extracted at the impinging point of the center of the crack. The only $A_{0}$ or Scolte modes were visible in these time domain signals. The $S_{0}$ modes were not observed even in the UWPI videos. In Figure 11(a), the arrival times of the Scolte modes in the water- and glycerin-immersed plates show time delays of $7.5 \mu \mathrm{s}$ and $9.5 \mu \mathrm{s}$, respectively, and the amplitudes of the Scolte modes waves in those conditions show decrease of $4.2 \mathrm{mV}$ and $10.1 \mathrm{mV}$, respectively, compared with the $A_{0}$ mode of the free plate.

Since the UWPI freeze-frames of Figures 5-7 show the moments of the maximum ultrasonic amplitudes at the crack location, the freezing times also imply the relative ultrasonic time-of-flights in the free plate and water- and glycerin-immersed plates, respectively. This information was used to calculate the experimental group velocities for the three different cases, which were determined at $2.35 \mathrm{~m} / \mathrm{ms}$, $1.82 \mathrm{~m} / \mathrm{ms}$, and $1.72 \mathrm{~m} / \mathrm{ms}$, respectively. This result was comparable to the theoretical results in the wave dispersion curves depicted in Figure 2 where the central frequency for each case was estimated based on fast Fourier transform and Hilbert transform as shown in Figure 11(b). The theoretical group velocities at the $44 \mathrm{kHz}$ for the free plate, $60 \mathrm{kHz}$ for the water immersion, and $44 \mathrm{kHz}$ for the glycerin immersion stood at $2.32 \mathrm{~m} / \mathrm{ms}, 1.90 \mathrm{~m} / \mathrm{ms}$, and $1.69 \mathrm{~m} / \mathrm{ms}$, respectively.

\section{Conclusion}

In this paper, a noncontact laser UPI system for submerged structural crack visualization was proposed. A $532 \mathrm{~nm}$ Q-switched solid-state laser and a $633 \mathrm{~nm}$ laser Doppler vibrometer were integrated into the system, for remote excitation and sensing, respectively. The tested specimen was an aluminum plate with dimensions of $400 \mathrm{~mm} \times 400 \mathrm{~mm} \times$ $3 \mathrm{~mm}$, which encompassed a back surface crack of $12 \mathrm{~mm} \times$ $1 \mathrm{~mm} \times 2 \mathrm{~mm}$ in the middle of the plate. Three cases were studied: free plate, water-immersed plate, and glycerinimmersed plate. 


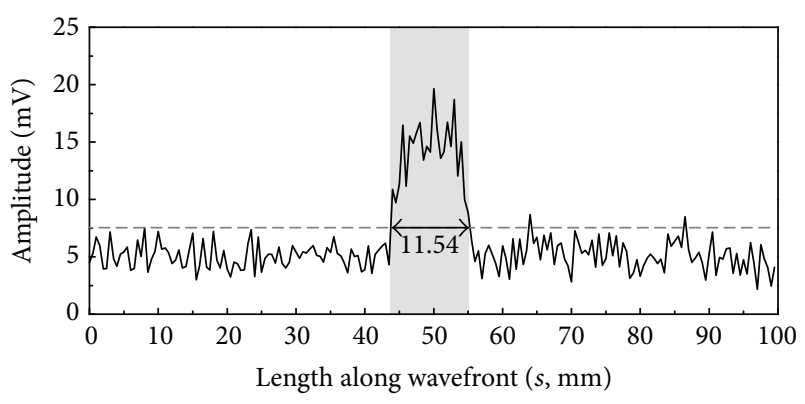

(a)

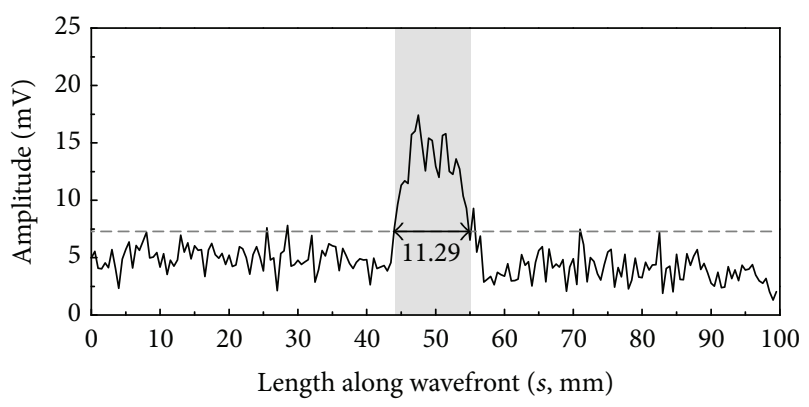

(b)

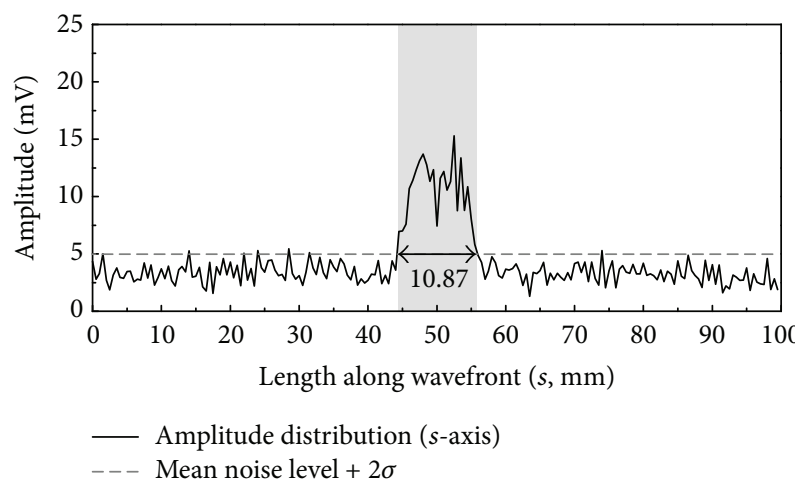

(c)

FIGURE 10: Crack length evaluation with the wave fields obtained in (a) the free plate (single scan), (b) the water-immersed plate (10 times scans), and (c) the glycerin-immersed plate (10 times scans).

First, theoretical wave dispersion curves were plotted, to understand the theoretical difference between the free and immersed plates. Then, wave propagation imaging for the submerged plates was successfully performed by the proposed system, where a laser mirror scanner and LDV were used for excitation laser scanning and noncontact sensing. The detected waves in the immersed plates showed delays in arrival time and reduction in amplitude, compared to the free plate, because of the surrounding liquid. In addition, the SNR was deteriorated in the submerged plates, because of the leaky Lamb wave. Therefore, the repeat scanning technique was incorporated into the system, to increase SNR up to a similar level to the single scan of the free plate and to prepare for real-world application that would involve more complex and thicker immersed structures. Finally, the proposed system

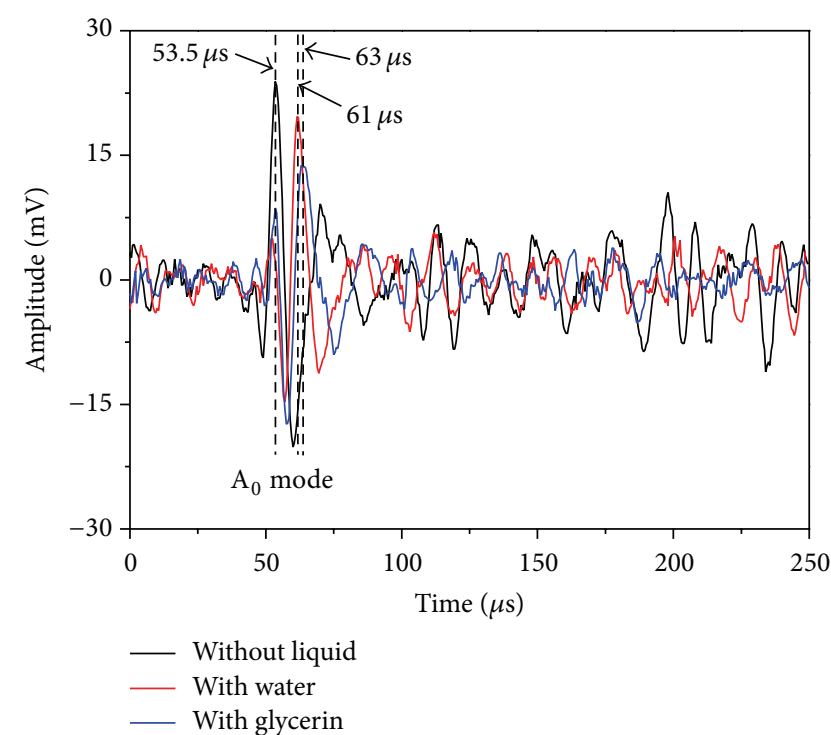

(a)
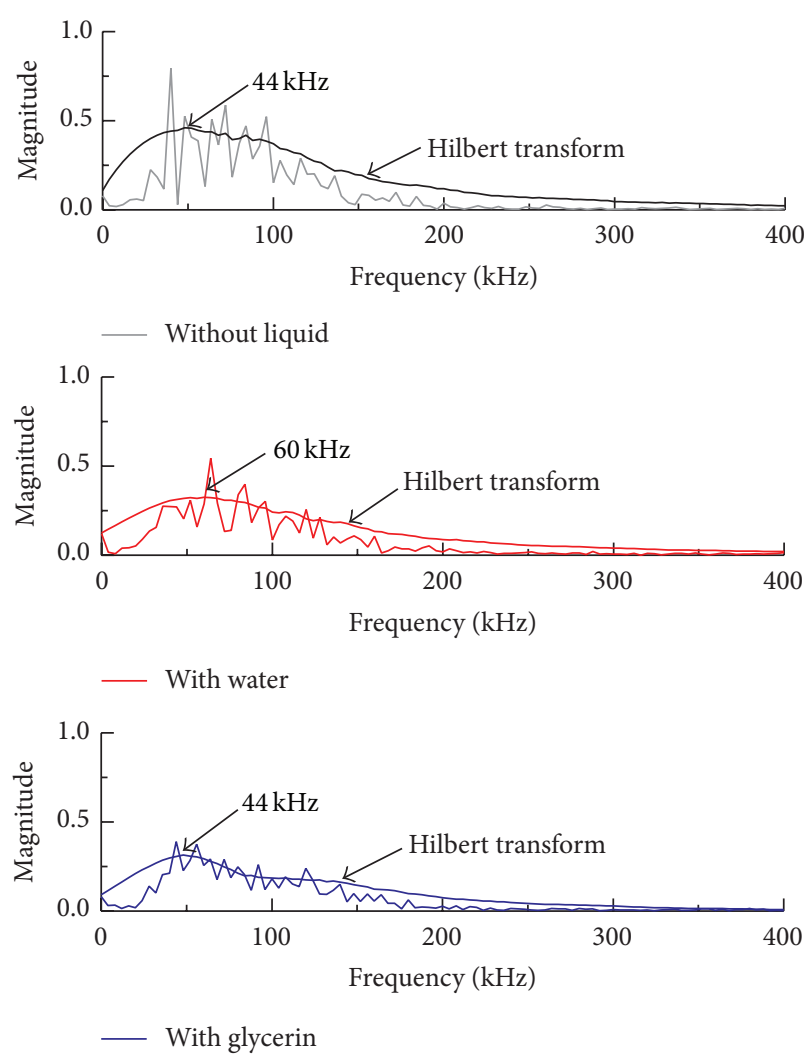

(b)

FIGURE 11: Signals extracted at the impinging point of the center of crack: (a) time domain and (b) frequency domain.

successfully visualized the wave propagation and showed damage evaluation capability for the submerged structural back surface crack. The $2 \mathrm{~mm}$ deep and $12 \mathrm{~mm}$ long back surface crack was evaluated by the proposed system as $11.29 \mathrm{~mm}$ in the water-immersed plate and $10.87 \mathrm{~mm}$ in the glycerin-immersed plate. 


\section{Conflict of Interests}

The authors declare that there is no conflict of interests regarding the publication of this paper.

\section{Acknowledgments}

This research was supported by the Leading Foreign Research Institute Recruitment Program (2011-0030065) and the Basic Science Research Program (2011-0010489) through the National Research Foundation of Korea, funded by the Ministry of Education, Science and Technology (2011-0030065) and by the university collaboration enhancement project of the Korea Aerospace Research Institute.

\section{References}

[1] W.-B. Na and T. Kundu, "Underwater pipeline inspection using guided waves," Journal of Pressure Vessel Technology, vol. 124, no. 2, pp. 196-200, 2002.

[2] R. Mijarez, P. Gaydecki, and M. Burdekin, "Flood member detection for real-time structural health monitoring of subsea structures of offshore steel oilrigs," Smart Materials and Structures, vol. 16, no. 5, pp. 1857-1869, 2007.

[3] J. Chen, Z. Su, and L. Cheng, "Identification of corrosion damage in submerged structures using fundamental anti-symmetric Lamb waves," Smart Materials and Structures, vol. 19, no. 1, Article ID 015004, 12 pages, 2010.

[4] C. Aristégui, M. J. S. Lowe, and P. Cawley, "Guided waves in fluid-filled pipes surrounded by different fluids," Ultrasonics, vol. 39, no. 5, pp. 367-375, 2001.

[5] P. Rizzo, J.-G. Han, and X.-L. Ni, "Structural health monitoring of immersed structures by means of guided ultrasonic waves," Journal of Intelligent Material Systems and Structures, vol. 21, no. 14, pp. 1397-1407, 2010.

[6] X. Xu, J. Goossens, G. Shkerdin, and C. Glorieux, "Effect of loading a plate with different liquids on the propagation of lamb-like waves studied by laser ultrasonics," IEEE Transactions on Ultrasonics, Ferroelectrics, and Frequency Control, vol. 55, no. 3, pp. 675-685, 2008.

[7] B. Pavlakovic and M. Lowe, DISPERSE: User's Manual Version 2.0.11, Imperial College, University of London, Non-Destructive Testing Laboratory, 2001.

[8] P. Moilanen, P. H. F. Nicholson, V. Kilappa, S. Cheng, and J. Timonen, "Measuring guided waves in long bones: modeling and experiments in free and immersed plates," Ultrasound in Medicine \& Biology, vol. 32, no. 5, pp. 709-719, 2006.

[9] F. B. Cegla, Ultrasonic waveguide sensors for fluid characterisation and remote sensing [Ph.D. thesis], University of London, 2006.

[10] J.-R. Lee and C.-Y. Yoon, "Development of an optical system for simultaneous ultrasonic wave propagation imaging at multipoints," Experimental Mechanics, vol. 50, no. 7, pp. 1041-1049, 2010.

[11] J. R. Lee, S. Y. Chong, N. Sunuwar, and C. Y. Park, "Repeat scanning technique to improve signal-to-noise ratio for laser ultrasonic propagation inspection for composite structures," Measurement Science and Technology, vol. 24, Article ID 085201, 2013.

[12] C. C. Chia, J.-R. Lee, and H.-J. Shin, "Hot target inspection using a welded fibre acoustic wave piezoelectric sensor and a laser-ultrasonic mirror scanner," Measurement Science and Technology, vol. 20, no. 12, Article ID 127003, 8 pages, 2009. 

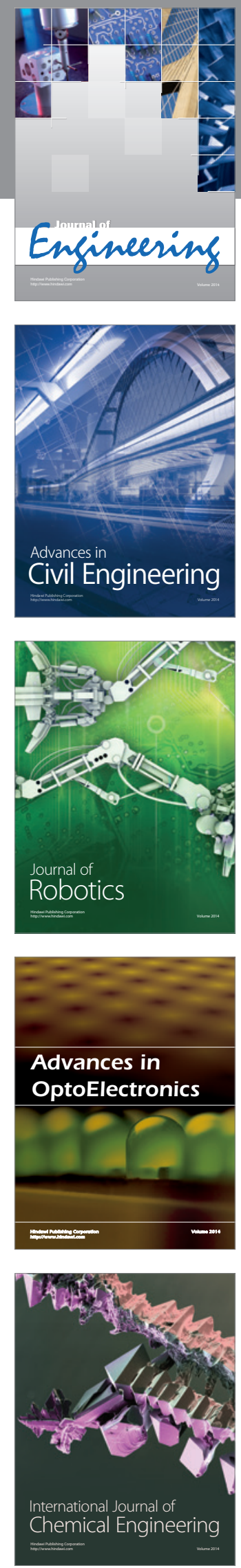

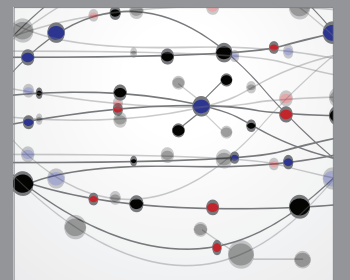

The Scientific World Journal
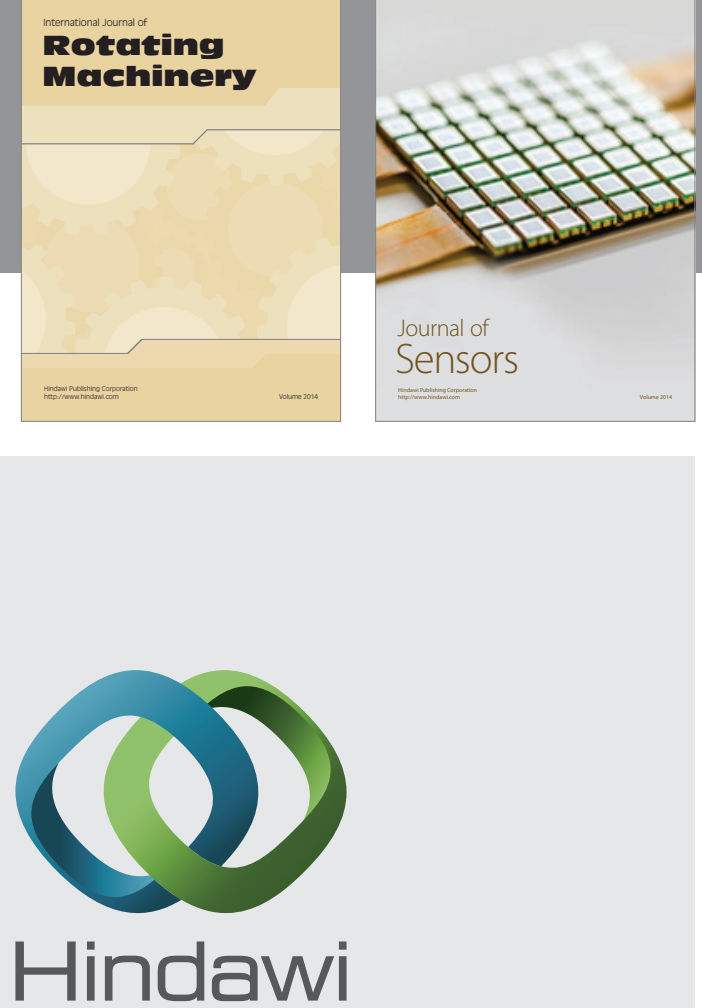

Submit your manuscripts at http://www.hindawi.com
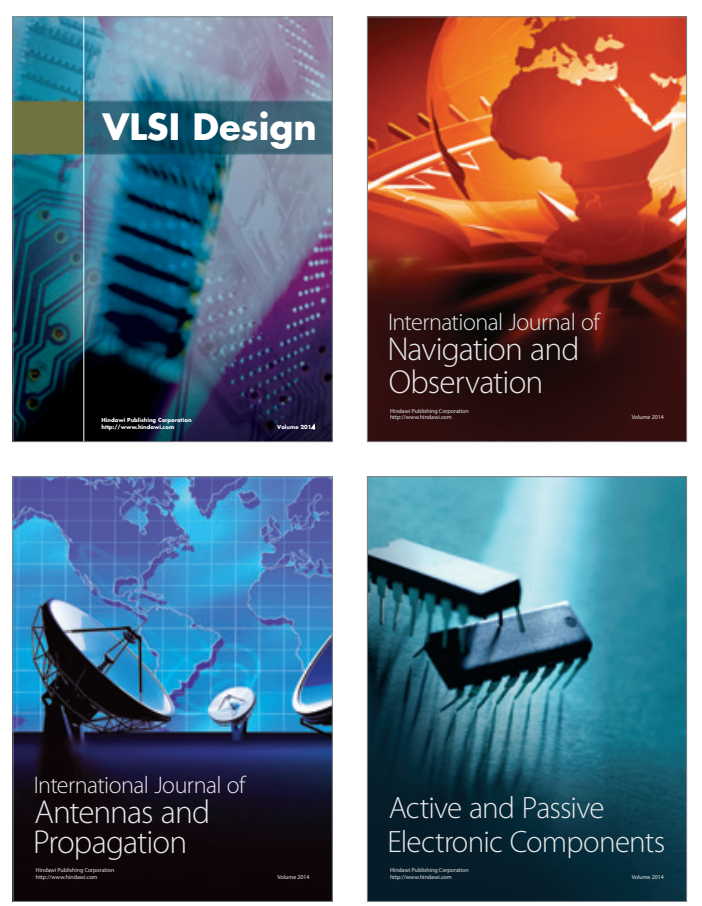
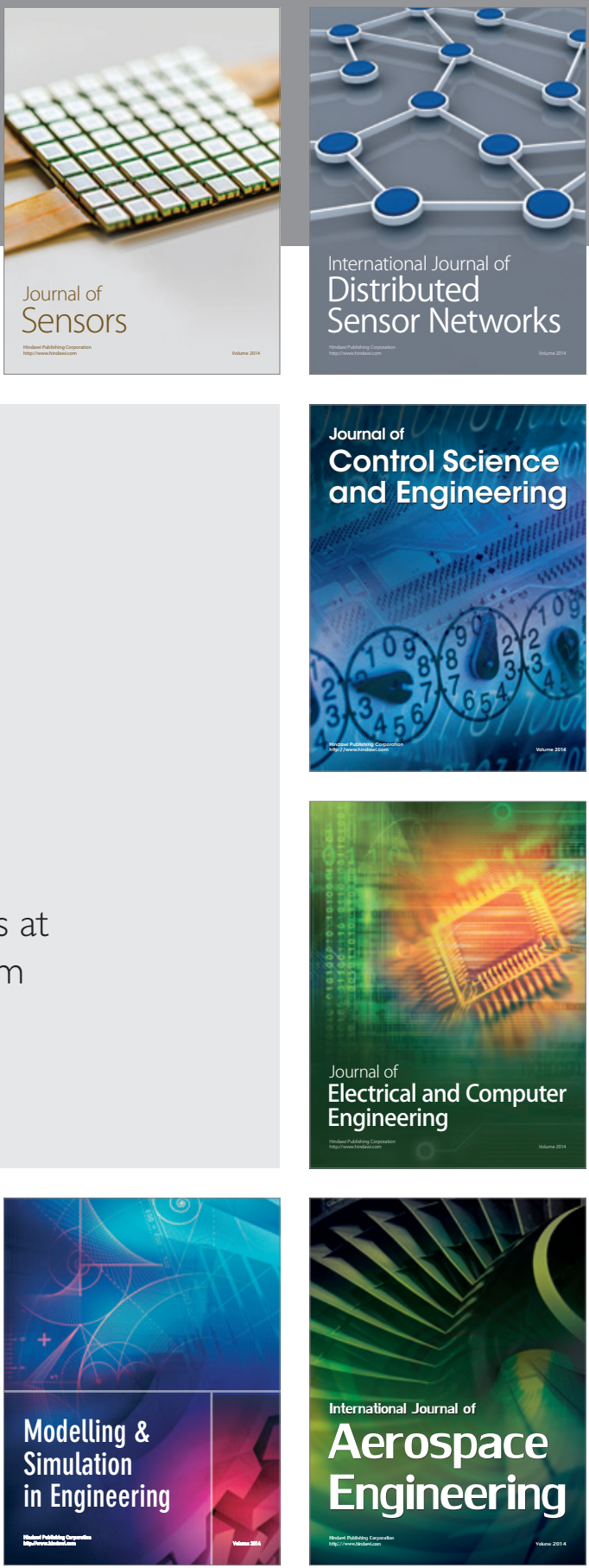

Journal of

Control Science

and Engineering
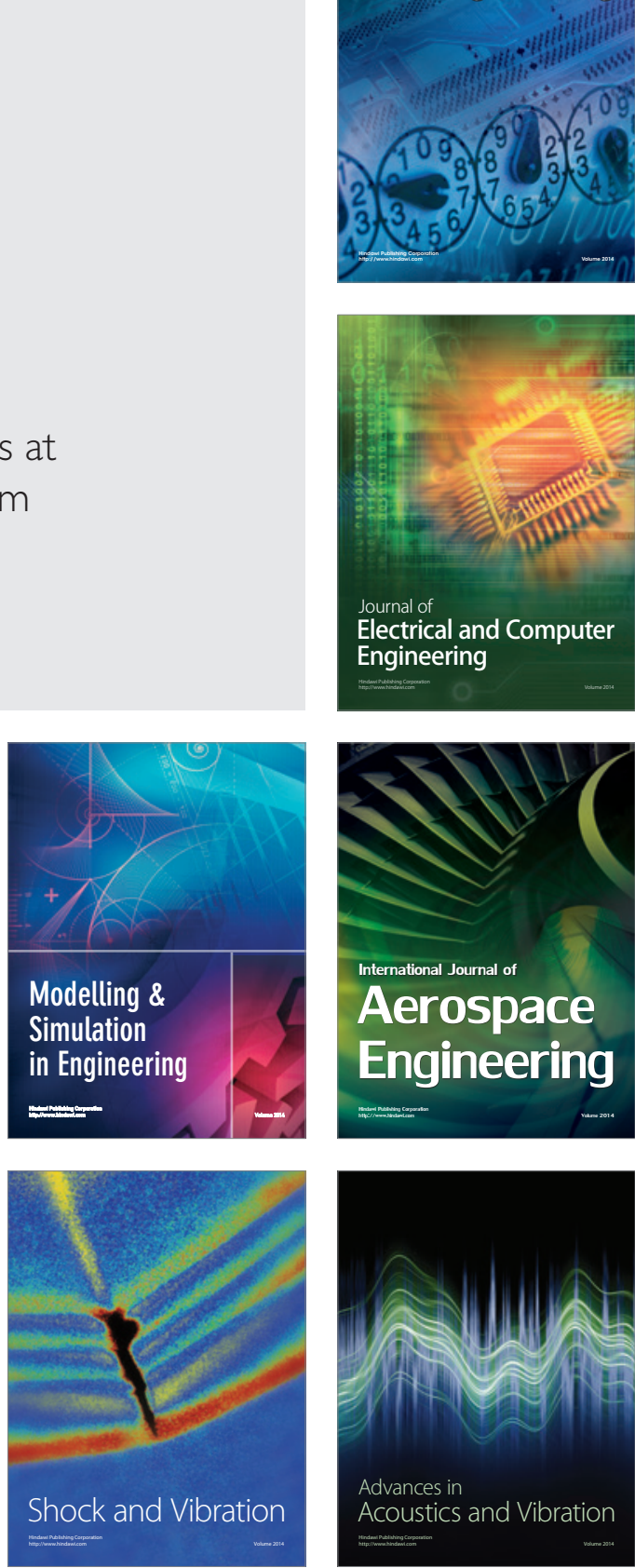